\title{
Caracterização do perfil epidemiológico da mortalidade por endocardite infecciosa na região Nordeste de 2010 - 2019
}

\author{
Characterization of the epidemiological profile of mortality from infectious endocarditis in \\ the Northeast region $2010-2019$
}

Caracterización del perfil epidemiológico de la mortalidad por endocarditis infecciosa en la región Nordeste 2010 - 2019

Soniely Nunes de Melo ${ }^{1 *}$, Bruna Rafaella Santos Torres ${ }^{1}$, Mônica Melo Gomes do Nascimento1, Antonio Fernando da Silva Xavier Júnior ${ }^{1}$, Wanessa Guimarães Rodrigues ${ }^{1}$, Rafael Belarmino de Souza Lima1, Nayara Sandriele Santana de Souza1, Luís Eduardo de França Barros Menezes².

\section{RESUMO}

Objetivo: Caracterizar o perfil de mortalidade por endocardite infecciosa na região nordeste entre 2010 e 2019. Métodos: Trata-se de um estudo epidemiológico do tipo transversal ecológico, com análise espacial dos casos notificados no Sistema de Informação de Agravos de Notificação (Sinan), software TabNet Win32 3.0. A amostra consiste em todos os óbitos por El aguda e subaguda na região nordeste no período compreendido entre 2010 e 2019, com análise das variáveis sexo e idade, sob classificação segundo a Classificação Internacional de Doenças em sua 10ª revisão - CID-10 (I33). Resultados: Identificaram-se 1335 casos diagnosticados, sendo 2019 o ano com o maior número de óbitos ( $n=178)$ e 2012 o ano com o menor número de óbitos $(n=120)$. Na análise realizada, a correlação de Pearson foi fortemente positiva para o sexo masculino $(r=0,97)$; raça parda $(r=0,87)$; escolaridade de 8 a 11 anos de estudo $(r=0,85)$; faixa etária entre 65 e 75 anos $(r=0,87)$; entre os casados $(r=0,82)$. Conclusão: Os resultados ratificam que esta doença é um desafio diagnóstico, tem alta mortalidade e está associada a complicações graves. Por ser um estudo retrospectivo, limita-se às informações disponíveis no Sinan.

Palavras-chave: Endocardite, Registros de mortalidade, Epidemiologia descritiva.

\section{ABSTRACT}

Objective: To characterize the mortality profile of infectious endocarditis in the northeast region between 2010 and 2019. Methods: An epidemiological study of the ecological cross-sectional type, with spatial analysis of cases notified in the Notifiable Diseases Information System (Sinan), TabNet Win32 3.0 software. The sample consists of all deaths from acute and subacute IE in the Northeast region in the period between 2010 and 2019, with analysis of gender and age variables, classified according to the International Classification of Diseases in its 10th revision - ICD-10 (I33). Results: 1,335 diagnosed cases were identified, 2019 being the year with the highest number of deaths $(n=178)$ and 2012 the year with the lowest number of deaths $(n=120)$. In the analysis performed, Pearson's correlation was strongly positive for males ( $r=0.97)$; brown race $(r=0.87)$; schooling from 8 to 11 years of schooling $(r=0.85)$; age group between 65 and 75 years $(r=0.87)$; married $(r=0.82)$. Conclusion: The results confirm that this disease is a diagnostic challenge, has a high mortality, and is associated with severe complications. Because it is a retrospective study, it is limited to the information available in Sinan.

Keywords: Endocarditis, Mortality registrations, Epidemiology describing.

${ }^{1}$ Centro Universitário Tiradentes (UNIT - AL), Maceió - AL. *E-mail: sonielymelomed@gmail.com

${ }^{2}$ Centro Universitário Uninovafapi (UNINOVAFAPI), Teresina - PI.

SUBMETIDO EM: 9/2021

ACEITO EM: 9/2021

PUBLICADO EM: 9/2021 


\section{RESUMEN}

Objetivo: Caracterizar el perfil de mortalidad por endocarditis infecciosa en la región noreste entre 2010 y 2019. Métodos: Se trata de un estudio epidemiológico ecológico transversal, con análisis espacial de los casos reportados en el Sistema de Información de Enfermedades Notificables (Sinan), TabNet Win32. Software 3.0. La muestra está compuesta por todas las defunciones por El aguda y subaguda en la región Nordeste en el período 2010-2019, con análisis de las variables de género y edad, clasificadas según la Clasificación Internacional de Enfermedades en su 10a revisión - CIE-10 (I33). Resultados: Se identificaron 1335 casos diagnosticados, siendo 2019 el año con mayor número de defunciones $(n=178)$ y 2012 el año con menor número de defunciones $(n=120)$. En el análisis realizado, la correlación de Pearson fue fuertemente positiva para los hombres $(r=0,97)$; raza marrón $(r=0,87)$; escolaridad de 8 a 11 años de estudio $(r=0,85)$; grupo de edad entre 65 y 75 años $(r=0,87)$; entre las personas casadas $(r=0,82)$. Conclusión: Los resultados confirman que esta enfermedad es un desafío diagnóstico, tiene una alta mortalidad y se asocia a graves complicaciones. Al tratarse de un estudio retrospectivo, se limita a la información disponible sobre Sinan.

Palabras clave: Endocarditis, Registros de mortalidad, Epidemiología descriptiva.

\section{INTRODUÇÃO}

A Endocardite Infecciosa (EI) caracteriza-se pela invasão de agentes infecciosos na superfície do endocárdio, principalmente valvar, ou em dispositivos implantados no coração, como próteses valvares, marca-passo e cardiodesfibriladores, produzindo inflamação local e frequentemente acúmulo de fibrinas e plaquetas (ACCORSI TAD, et al., 2016). Trata-se de uma patologia com incidência anual variando de 1,5 a 15 casos por 100.000 habitantes e embora relativamente infrequente, apresenta alta taxa de mortalidade, variando de 20 a $25 \%$, uma vez que mais de um terço dos pacientes falecem no primeiro ano após o diagnóstico (CUERVO G, et al., 2021; SOBREIRO DI, et al., 2019).

O perfil epidemiológico evolutivo da El, incluindo sua apresentação clínica variável, o espectro dos microrganismos envolvidos, as características intrínsecas dos pacientes e o uso crescente de materiais protéticos e Dispositivos Intracardíacos (DIC) a mantém como um desafio diagnóstico (GROSSMAN GB e CARREIRA LFT, 2019).

Em relação à sua patogênese, é sabido que o endotélio valvar normal é resistente à colonização por bactérias, por isso cerca de 70\% das El ocorrem em portadores de doenças cardíacas prévias, sendo a causa mais comum o turbilhonamento sanguíneo que ocorre em valvas previamente lesadas (ACCORSI TA, et al., 2016; MADEIRA MP, 2018). Uma vez lesado, o endotélio cursa com formação de vegetação trombótica não bacteriana, devido à agregação de plaquetas, trombos vermelhos e fibrina. Posteriormente, há a aderência de bactérias circulantes na corrente sanguínea, resultando em proliferação da vegetação infectada e, consequentemente, agravamento da lesão valvar (CAHILL TJ, et al., 2017).

Assim, vários microrganismos podem causar endocardite, sendo os estreptococos os agentes mais comuns e os estafilococos os mais associados a complicações e a infecção sobre dispositivos cardíacos (VIEIRA PM, et al., 2020). É válido ressaltar que há diferença de prevalência entre os diversos microrganismos associados à El, de acordo com o subgrupo de classificação desta ACCORSI TAD, et al., 2016).

Quanto ao diagnóstico de El, este pode ser extremamente difícil se o médico não suspeitar da doença, pois essa apresenta inúmeras manifestações clínicas, que aparecem conforme a doença de base do paciente, o agente etiológico e o tempo de instalação do tratamento (MARQUES A, et al., 2019).

Todos os sinais e sintomas sugestivos são inespecíficos, como febre, presente em até $90 \%$ dos pacientes, cefaléia, sudorese noturna, dispneia, dor abdominal e mialgias (ACCORSI TAD, et al., 2016). Além desses, existem sinais e sintomas clínicos mais correlacionados com a doença, embora menos frequentes, como petéquias, hemorragias em cunha e subungueais, nódulos de Osler e lesões de Janeway (KARCHMER AW, et al., 2012). 
Atrelado às manifestações clínicas, as alterações laboratoriais são em sua maioria inespecíficas e sem correlação significativa com o diagnóstico, sendo obrigatória a utilização de métodos diagnósticos apropriados, como hemoculturas e ecocardiograma (MARQUES A, et al., 2019). Acredita-se que o emprego de modalidades de imagem não invasivas poderia trazer uma boa resposta diagnóstica, contudo sua eficácia ainda não é clara (GOMES A, et al., 2016). Portanto, os achados clínicos, biológicos e ecocardiográficos, associadas a hemoculturas e sorologias são organizados em critérios maiores e menores, o que ficou conhecido como Critérios de Duke modificados, a fim de garantir o diagnóstico e terapêutica precoces, uma vez que esses são necessários para reduzir essa elevada mortalidade (SOBREIRO DI, et al., 2019).

Dessa forma, o médico generalista precisa ter conhecimento dos principais fatores que interferem e aumentam a chance do surgimento da $\mathrm{El}$, os quais englobam valvopatias, como doença reumática, alterações degenerativas e prolapso mitral com insuficiência valvar, prótese valvar, cardiopatia congênita cianótica e o uso de dispositivos implantados ou de drogas intravenosas (MARQUES A, et al., 2019). Ademais, não se deve descartar 0 atendimento multidisciplinar para o manejo dessa enfermidade, que pode envolver a expertise de cardiologistas, cirurgiões cardiotorácicos, especialistas em doenças infecciosas, radiologistas e neurologistas (HUBERS SA, et al., 2020).

Em relação ao tratamento com antibióticos, mesmo não reduzindo os índices de mortalidade, proporcionou aumento da sobrevida (LAMAS CC, 2020). Os profissionais da área devem estar atentos há necessidade de divergentes opções de tratamento, como antibióticos mais novos e regimes de terapia de combinação, uma vez que nos últimos anos observou-se uma maior resistência dos microrganismos, principalmente 0 Staphylococcus aureus (WANG A, et al., 2018). O tratamento cirúrgico, por sua vez, está se estabelecendo como um fator protetor para mortalidade, sendo necessário em cerca de metade dos pacientes, independente da duração do tratamento com antibióticos (LAMAS CC, 2020).

De acordo com a Diretriz Brasileira de Valvopatias (2011) e as Diretrizes da European Society of Cardiology (2015), as recomendações para a profilaxia estão bem estabelecidas e envolvem principalmente esquemas de profilaxia com antibióticos em procedimentos invasivos, odontológicos ou não, em pacientes com fatores de risco para evolução com El: pacientes com válvula protética, pacientes com El anterior e/ou pacientes com doença cardíaca cianótica (TARASOUTCHI F, et al., 2011; HABIB G, et al., 2015).

Dessa forma, o presente estudo objetiva analisar o perfil epidemiológico dos óbitos por endocardite infecciosa aguda e subaguda na região Nordeste entre os anos de 2010 e 2019.

\section{MÉTODOS}

O presente trata-se de um estudo descritivo de caráter qualitativo e quantitativo. Para isto, foi realizado um estudo epidemiológico do tipo transversal ecológico. Foram utilizados dados secundários de domínio público disponíveis no site do DATASUS. A coleta de informações estatísticas ocorreu através do banco de dados disponíveis no Departamento de Informática do Sistema Único de Saúde, coletados no Sistema de Informação de Mortalidade (SIM), software TabNet Win32 3.0.

A amostra consiste em todos os óbitos por El aguda e subaguda na região Nordeste brasileira no período compreendido entre os anos de 2010 e 2019, com análise das variáveis sexo e idade, sob código I33 segundo a Classificação Internacional de Doenças em sua 10ª revisão -CID-10.

As variáveis dependentes foram: número de óbitos por sexo, faixa etária, raça e região de saúde, estado civil e escolaridade. A variável independente foi o ano de óbito. Foi utilizada a estatística descritiva, por meio da descrição tabular e gráfica, para apresentação dos dados. As análises dos dados foram realizadas através do software Microsoft ${ }^{\circledR}$ Excel, através da correlação de Pearson. As tendências significativas foram assumidas quando apresentarem valores de $R^{2}$ maior que 0,399 .

Tendo como base o coeficiente de correlação $(R)$ e a relação representada na Tabela 1, correlações significativas foram observadas entre a variável independente e as variáveis dependentes, dentre suas respectivas subdivisões. 
Tabela 1 - Valores do coeficiente de correlação de Pearson ( $r$ ).

\begin{tabular}{cc}
\hline Coeficiente de correlação & Correlação \\
$r=1$ & Perfeita Positiva \\
$0,8<=r<1$ & Forte Positiva \\
$0,5<=r<0,8$ & Moderada Positiva \\
$0,1<=r<0,5$ & Fraca Positiva \\
$0<r<0,1$ & Ínima Positiva \\
$r=0$ & Nula \\
$(-0,1)<r<0$ & Ínfima Negativa \\
$(-0,5)<r<=(-0,1)$ & Fraca Negativa \\
$(-0,8)<r<=(-0,5)$ & Moderada Negativa \\
$(-1)<r<=(-0,8)$ & Forte Negativa \\
$r=(-1)$ & Perfeita Negativa \\
\hline
\end{tabular}

Fonte: Melo SN, et al., 2021; baseado no Coeficiente de Correlação Linear de Pearson.

Considerando que este estudo não envolve diretamente a coleta de dados com seres humanos, foram utilizados dados de domínio público, o que garante, portanto, a não identificação do sujeito pesquisado. Este estudo não necessitou de aprovação do Comitê de Ética em Pesquisa, em conformidade com a Resolução do Conselho Nacional de Saúde, № 466 de 2012, além de respeitar as especificidades éticas da Resolução no 510 de 7 de abril de 2016.

Para a fundamentação teórica foram selecionados artigos científicos, livros e informações das organizações de referência mundial sobre o tema. As buscas foram realizadas nas bases de dados do Scielo e do PubMed, com periodicidade nos últimos 10 anos, em português e em inglês. Como critérios de exclusão, estabeleceu-se a retirada de artigos que apresentavam pouca ou nenhuma informação relevante sobre 0 tema, ou que não possuíam informações adicionais aos artigos já selecionados.

\section{RESULTADOS}

De acordo com esses dados coletados, entre janeiro de 2010 e dezembro de 2019, registrou-se 1335 casos de óbitos por endocardite infecciosa aguda e subaguda através dos critérios de Duke notificados no SIM. Verificou-se que dos anos analisados, 2019 foi o ano com o maior número de óbitos $(n=178)$ e 2012 o ano com o menor número de óbitos $(n=120)$. Esses dados ainda revelaram uma semelhança no número de óbitos entre $o$ ano de 2010 e 2011 ( $n=135$ ) (Tabela 2).

Dos pacientes analisados, $70,3 \%$ são do sexo masculino e $42,9 \%$ do sexo feminino, sendo todos brasileiros, na região Nordeste. A faixa etária, independente de sexo, com maior número de casos notificados no presente estudo foi de 55 a 64 anos, com um total de 276 casos.

Os dados também foram classificados, de acordo com os critérios do Censo Brasileiro, em brancos $(n=482)$, pretos $(n=139)$ e pardos $(n=811)$. Não houve análise da raça indígena e amarela, pela ausência de dados na coleta do DATASUS. Além disso, os dados referentes ao estado civil mostraram que 566 indivíduos estavam casados e 495 estavam solteiros.

$\mathrm{Na}$ análise realizada, percebeu-se que a correlação entre o número de óbitos por ano e a variável sexo foi fortemente positiva para o sexo masculino $(r=0,97)$ e moderadamente positiva para o sexo feminino $(r=0,65)$.

Considerando a variável raça, notou-se correlação fortemente positiva com a raça parda $(r=0,87)$, moderadamente positiva com a raça branca $(r=0,75)$ e fracamente positiva com a raça preta $(r=0,43)$.

Em relação à variável escolaridade, mostrou-se uma correlação fortemente positiva com indivíduos com 8 a 11 anos de estudo $(r=0,85)$; moderadamente positiva para escolaridade de 1 a 3 anos $(r=0,73)$, para nenhuma escolaridade $(r=0,63)$ e para escolaridade de 4 a 7 anos $(r=0,51)$; fracamente positiva para indivíduos com 12 anos ou mais de escolaridade $(r=0,48)$.

A correlação bivariável entre o número de óbitos por ano e a faixa etária revelou fortemente positiva em indivíduos entre 65 e 75 anos ( $r=0,87)$; moderadamente positiva entre 35 e 44 anos ( $r=0,60)$; entre 45 e 54 anos ( $r=0,77)$; entre 55 e 64 anos $(r=0,67)$; e acima de 75 anos $(r=0,69)$. 
Foi possível perceber a fraca correlação entre o número de óbitos por ano e a faixa etária que varia entre menores de 1 ano e 34 anos de idade, sendo fracamente negativa em menores de 1 ano $(r=-0,013)$; em indivíduos de 1 a 4 anos ( $r=-0,321)$; fracamente positiva em indivíduos entre 5 e 14 anos ( $r=0,23)$; moderadamente negativa na faixa etária de 15 a 24 anos $(r=-0,60)$; fracamente positiva na faixa de 25 a 34 anos $(r=0,29)$. Na correlação bivariável óbitos por ano e estado civil, obteve-se fortemente positiva entre os casados $(r=0,82)$ e moderadamente positiva entre os solteiros $(r=0,62)$.

Os resultados (Tabela 2) evidenciam que $94,2 \%$ da variação no número de óbitos por ano é explicada por variação no sexo masculino e cerca de $43 \%$ por variação no sexo feminino. Correlações significativas foram observadas nas raças branca $\left(R^{2}=57,2 \%\right)$ e parda $\left(R^{2}=76,7 \%\right)$.

No que tange à variável escolaridade (Tabela 3), destaca-se que cerca de $40 \%$ da variação de óbitos por ano seja explicada pelo número de indivíduos com nenhuma escolaridade, 53,8\% por escolaridade de 1 a 3 anos e $72,3 \%$ por escolaridade de 8 a 11 anos.

Foram observadas diferenças significativas quanto à variável faixa etária (Tabela 4), determinando relação significante as faixas etárias a partir de 45 anos de idade: 45 a 54 anos $\left(R^{2}=60,0 \%\right) ; 55$ a 64 anos $\left(R^{2}=45,8 \%\right)$; 65 a 74 anos $\left(R^{2}=76,8 \%\right) ; 75$ anos ou mais $\left(R^{2}=48,2 \%\right)$.

Tem-se que $68,8 \%$ da variação no número de óbitos por ano é acompanhada pela variação no estado civil casado. Assim como a correlação com a variável raça reflete que $57,2 \%$ da variação na mortalidade anual é determinada pela variação da raça branca, enquanto $76,74 \%$ é pela variação da raça parda.

Tabela 2 - Número de óbitos por ano por Endocardite Infecciosa segundo suas variáveis dependentes.

\begin{tabular}{ccccccccc}
\hline Ano de óbitos & $\begin{array}{c}\text { Número de } \\
\text { óbitos por } \\
\text { ano }\end{array}$ & $\begin{array}{c}\text { Sexo } \\
\text { Feminino }\end{array}$ & $\begin{array}{c}\text { Sexo } \\
\text { Masculino }\end{array}$ & $\begin{array}{c}\text { Raça } \\
\text { branca }\end{array}$ & $\begin{array}{c}\text { Raça } \\
\text { preta }\end{array}$ & $\begin{array}{c}\text { Raça } \\
\text { parda }\end{array}$ & Solteiro & Casado \\
\hline 2010 & 135 & 51 & 84 & 42 & 17 & 67 & 51 & 49 \\
2011 & 135 & 53 & 82 & 57 & 13 & 56 & 47 & 56 \\
2012 & 120 & 49 & 71 & 35 & 11 & 67 & 40 & 43 \\
2013 & 130 & 58 & 72 & 41 & 11 & 71 & 41 & 53 \\
2014 & 145 & 62 & 83 & 43 & 13 & 84 & 50 & 53 \\
2015 & 160 & 59 & 101 & 44 & 17 & 86 & 51 & 51 \\
2016 & 176 & 69 & 107 & 58 & 12 & 99 & 58 & 66 \\
2017 & 157 & 57 & 100 & 48 & 7 & 94 & 59 & 53 \\
2018 & 177 & 60 & 117 & 57 & 18 & 91 & 50 & 65 \\
2019 & 178 & 56 & 122 & 57 & 20 & 96 & 48 & 77 \\
\hline$R^{2}$ Pearson & & 0,43 & 0,94 & 0,57 & 0,18 & 0,76 & 0,39 & 0,68 \\
\hline
\end{tabular}

Fonte: Melo SN, et al., 2021; dados extraídos do SIM/SUS- DATASUS. Ministério da Saúde, 2021.

Tabela 3 - Número de óbitos por ano por Endocardite Infecciosa segundo a variável escolaridade.

\begin{tabular}{cccccc}
\hline Ano de óbitos & Nenhuma & $\mathbf{1}$ a 3 anos & $\mathbf{4}$ a 7 anos & $\mathbf{8}$ a 11 anos & $\mathbf{1 2}$ ou mais anos \\
\hline 2010 & 7 & 28 & 29 & 14 & 8 \\
2011 & 13 & 21 & 30 & 24 & 16 \\
2012 & 12 & 21 & 26 & 28 & 11 \\
2013 & 12 & 30 & 22 & 21 & 8 \\
2014 & 15 & 30 & 21 & 24 & 6 \\
2015 & 10 & 39 & 28 & 34 & 12 \\
2016 & 15 & 45 & 30 & 40 & 11 \\
2017 & 21 & 32 & 34 & 33 & 11 \\
2018 & 21 & 33 & 30 & 41 & 16 \\
2019 & 19 & 31 & 31 & 40 & 16 \\
\hline$R^{2}$ Pearson & 0,40 & 0,53 & 0,26 & 0,72 & 0,23 \\
\hline
\end{tabular}

Fonte: Melo SN, et al., 2021; dados extraídos do SIM/SUS- DATASUS. Ministério da Saúde, 2021. 
Tabela 4 - Número de óbitos por ano por Endocardite Infecciosa segundo a variável faixa etária.

\begin{tabular}{ccccccc}
\hline Ano de óbito & $\begin{array}{c}\mathbf{2 5} \text { a } \mathbf{3 4} \\
\text { anos }\end{array}$ & $\begin{array}{c}\mathbf{3 5} \text { a } \mathbf{4 4} \\
\text { anos }\end{array}$ & $\begin{array}{c}\mathbf{4 5} \text { a } \mathbf{5 4} \\
\text { anos }\end{array}$ & $\begin{array}{c}\mathbf{5 5} \text { a } \mathbf{6 4} \\
\text { anos }\end{array}$ & $\begin{array}{c}\mathbf{6 5} \text { a } \mathbf{7 4} \\
\text { anos }\end{array}$ & $\begin{array}{c}\mathbf{7 5} \text { anos ou } \\
\text { mais }\end{array}$ \\
\hline 2010 & 18 & 18 & 16 & 18 & 24 & 16 \\
2011 & 13 & 16 & 15 & 24 & 23 & 16 \\
2012 & 17 & 10 & 22 & 20 & 13 & 25 \\
2013 & 11 & 20 & 13 & 27 & 23 & 22 \\
2014 & 8 & 18 & 29 & 33 & 23 & 16 \\
2015 & 13 & 26 & 25 & 29 & 29 & 22 \\
2016 & 24 & 19 & 33 & 30 & 31 & 28 \\
2017 & 13 & 18 & 27 & 30 & 32 & 21 \\
2018 & 15 & 23 & 29 & 38 & 30 & 34 \\
2019 & 15 & 19 & 28 & 27 & 41 & 35 \\
\hline $\mathrm{R}^{2}$ Pearson & 0,08 & 0,37 & 0,60 & 0,45 & 0,76 & 0,48
\end{tabular}

Fonte: Melo SN, et al., 2021; dados extraídos do SIM/SUS- DATASUS. Ministério da Saúde, 2021.

\section{DISCUSSÃO}

Neste estudo foram encontrados 1513 óbitos no período em análise. Os resultados obtidos demonstraram correlações mais significativas do número de óbitos de endocardite infecciosa aguda e subaguda por ano no sexo masculino, na raça parda, entre os casados, no nível de escolaridade de 8 a 11 anos e na faixa etária de 65 a 74 anos de idade.

A amostra foi representativa dos dados disponíveis no DATASUS, a partir do departamento do SIM, que correspondem à população padrão atendida nos serviços públicos de saúde da região Nordeste, a qual é responsável por cerca de $18 \%$ da mortalidade por El no Brasil, no período estudado.

O perfil sociodemográfico observado concorda com estudos realizados, os quais revelam que a endocardite incide globalmente em uma proporção de dois homens para cada mulher, já sendo possível também comparar, por esse estudo, que a média de idade dos pacientes aumentou gradualmente (ZIPES DP, et al., 2013).

A faixa etária média do estudo foi de 55 a 64 anos, semelhante ao estudo realizado na Itália em 2013, no qual a idade média foi de 57 anos. A idade média foi diferente quando comparada aos estudos realizados em Belém do Pará, que foi de 46 anos, e no Ceará que foi de 45 anos (HOLANDA LS, et al., 2016; MADEIRA MP, 2018).

Os dados desta pesquisa corroboram com estudos recentes quanto à faixa etária que evidenciaram mudanças significativas nas características epidemiológicas da El nas últimas décadas. Observa-se que, conforme as intervenções médicas tornaram-se cada vez mais realizadas em pacientes mais velhos, concomitantemente há um aumento na incidência da doença por dispositivos eletrônicos implantados cardíacos (WANG A, et al. 2018).

Segundo Holanda, et al. (2016), a incidência de El por idade aumentou de 5 casos por 100.000 pessoas/ano entre indivíduos $<50$ anos de idade para 15 a 30 casos por 100.000 pessoas/ano entre indivíduos da sexta até a oitava década de vida. Em relação à população geral, observou-se um risco 4,6 maior de El em pacientes idosos, principalmente aqueles que apresentam comorbidades prévias, principalmente diabetes (20\%), insuficiência renal crônica (14\%) e anemia (10\%) (SOBREIRO DI, et al., 2019).

A El também está cada vez mais relacionada a portadores de prótese valvares, cateteres vasculares, dispositivos eletrônicos implantáveis, como marcapasso e cardiodesfibriladores, e novos dispositivos cirúrgicos, como endopróteses valvares implantadas por cateter (COELHO AP, et al., 2018).

A partir de 2012, o número de óbitos elevou-se anualmente e, de acordo com os critérios de notificação da doença, o ano de 2019 totalizou o maior número de óbitos, representando uma crescente, a qual pode ser atribuída à maior facilitação na notificação, reduzindo-se os casos anteriormente subnotificados, e/ ou à 
letalidade da doença ainda sobrepõe os esforços da medicina para combatê-la. Esse aumento anual crescente pode refletir uma futura mudança no status de doença ainda que relativamente infrequente, com cerca de 3 a 10 casos por 100.000 pacientes/ano (SOBREIRO DI, et al., 2019).

Nos artigos científicos selecionados para embasamento deste estudo, não foram encontrados resultados analisando as variáveis de escolaridade e estado civil. No entanto, no presente estudo a escolaridade tem sido utilizada como indicador da situação socioeconômica. Assumindo-se que $72 \%$ da variação de número de óbitos é acompanhada pela variação entre indivíduos com 8 a 11 anos de estudo, o que concorda com o perfil de pacientes que conseguem ter acesso ao serviço de saúde, de acordo com o DATASUS. Outrossim, entre os estados civis analisados, destacaram-se os casados com coeficiente de determinação maior que $68 \%$.

Torna-se ainda relevante analisar comparativamente estudos realizados e publicados nos últimos anos em relação ao Nordeste, uma vez que possibilita mensurar a realidade da El na região. Sendo assim, verificouse em estudo realizado em hospital do Ceará, $25 \%$ de mortalidade por El, sendo revistos 12 casos (MADEIRA MP, 2018). No Rio Grande do Norte, por sua vez, houve uma taxa de óbito de 17,4\%, em uma análise de 110 casos da doença (MACHADO FCA e FERREIRA MAF, 2013). Comparativamente, a taxa de óbitos na região não supera dados do Sudeste, como é possível analisar em estudos realizados em hospitais do Rio de Janeiro, com variação de $22 \%$ a $50 \%$ de mortalidade (FRANCISCHETTO O, et al., 2014).

Para verificar fatores modificáveis, estabelecer o padrão de tratamento para cada paciente e consequentemente melhorar o seu desfecho clínico, é imprescindível avaliar os preditores de eventos fatais. Verificou-se que embora os critérios modificados da Universidade de Duke tenham sensibilidade aproximada de $80 \%$, apresentam uma menor precisão diagnóstica para o diagnóstico precoce na prática clínica, sendo assim, o diagnóstico da El tornou-se um desafio ao médico (SOBREIRO DI, et al., 2019; PINHEIRO IL, et al., 2018).

Essa dificuldade diagnóstica resulta da possibilidade de resultados negativos nos critérios de Duke, uma vez que a ecocardiografia pode ser normal ou inconclusiva, como ocorre em até $30 \%$ dos casos, e as hemoculturas podem ser negativas em cerca de $2 \%$ a $20 \%$ dos casos de endocardite (AFONSO L, et al., 2017; LIESMAN RM, et al., 2017).

Além disso, existem fatores confundidores, como congestão pulmonar e alteração em exames de urina, que podem fazer com que pacientes com El sejam erroneamente diagnosticados (HABIB G, 2015). Por isso, na prática clínica, a El sempre deve ser uma hipótese diagnóstica, especialmente em pacientes com fatores predisponentes e de risco conhecidos, como valvopatias, próteses valvares, dispositivos cardíacos eletrônicos implantáveis, cateter de longa permanência, uso de droga injetável, entre outros.

Uma vez estabelecido o diagnóstico, a identificação rápida dos casos com maior risco de morte influencia diretamente na evolução da doença, podendo melhorar seu prognóstico, o qual depende de quatro fatores principais: características particulares do paciente, presença de complicações cardíacas, o microrganismo infectante e os achados ao ecocardiograma. É sabido que na presença de pelo menos três desses fatores o risco de mortalidade alcança a margem de $79 \%$, somando-se a um alto grau de comorbidades, o desfecho hospitalar torna-se de maior gravidade (HABIB G, et al., 2015).

A avaliação prognóstica na admissão embasada em parâmetros clínicos, microbiológicos e ecocardiográficos simples devem ser utilizados para condução do caso para a melhor abordagem inicial das variáveis significativas na mortalidade da El facilita a identificação dos pacientes com maior risco de morte para os quais o nível de cuidados deve ser intensificado. Entre as limitações do presente estudo, as informações foram limitadas aos dados notificados no DATASUS, portanto a existência de números subnotificados impossibilitou estimar com maior profundidade o impacto da El em outras importantes variáveis da saúde.

Portanto, considerando-se a amostragem usada neste estudo e a correlação linear estabelecida, não é possível extrapolar as conclusões para outros intervalos de dados. O fato deste estudo ter sido realizado através do banco de dados do SUS, permite inferir comportamento semelhante desta amostra específica em relação aos estados da região Nordeste. 


\section{CONCLUSÃO}

Os resultados obtidos demonstraram maiores correlações do número de óbitos de endocardite infecciosa aguda e subaguda por ano no sexo masculino, na raça parda, entre os casados, no nível de escolaridade de 8 a 11 anos e na faixa etária de 65 a 74 anos de idade. Ainda com base nesse estudo, a avaliação das taxas de mortalidade e de preditores de eventos fatais ratifica a importância de identificar clinicamente os casos suspeitos precocemente e estabelecer o padrão de tratamento, a fim de melhorar ainda mais os desfechos apesar da gravidade da doença. Por conseguinte, estudos como este se fazem necessários para a avaliação das notificações em saúde pública e alerta para construção de medidas preventivas que combatam a evolução da endocardite infecciosa.

\section{REFERÊNCIAS}

1. ACCORSI TAD, et al. Valvopatias e Endocardite Infecciosa. In: Clínica Médica [2ed. ampl. rev.]. Manole, 2016.

2. AFONSO L, et al. Echocardiography in infective endocarditis: state of the art.Current cardiology reports, 2017; 19(12): 1-13.

3. BRAVO DPL, et al. Tratamento da endocardite infecciosa com troca valvar. Cadernos da Medicina-UNIFESO, 2018; $1(1)$.

4. CAHILL TJ, et al. Desafios na endocardite infecciosa. Journal Ofthe American College of Cardiology, 2017; 69(3): 325344.

5. COELHO AP, et al. Tratamento da endocardite infecciosa com troca valvar. Revista Caderno de Medicina, 2018; 1(1).

6. CUERVO G, et al. Desafios atuais na gestão da endocardite infecciosa. Med dianteiro (Lausanne), 2021; 8(641243).

7. FERRARIS $L$, et al. Profile of infective endocarditis observed from $2003-2010$ in a single center in Italy. BMC Infectious Diseases, Milão - Itália, 2013; 13 (545):1-9.

8. FRANCISCHETTO O, et al. Endocardite infecciosa associada aos cuidados de saúde: série de casos em hospital de referência entre 2006 e 2011. Arq. Bras. Cardiol, 2014; 103 (4).

9. GOMES A, et al. Diagnostic value of imaging in infective endocarditis: a systematic review. Lancet Infect Dis; 2017; 17(1): 1-14.

10. GONÇALVES IN. Profilaxia antimicrobiana em medicina dentária: uma revisão da literatura. Dissertação (Mestrado integrado em Medicina Dentária) - Instituto Superior de Ciências da Saúde Egaz Moniz. 2013; 63 p.

11. GROSSMAN GB, CARREIRA LTF. Papel do PET/CT no diagnóstico da Endocardite Infecciosa. Arq Bras Cardiol: Imagem Cardiovasc, 2019; 32(3): 209-213.

12. HABIB G, et al. Guidelines For The Management Of Infective Endocarditis: The Task Force For The Management Of Infective Endocarditis Of The European Society Of Cardiology. European Heart Journal, 2015; 3075-3128.

13. HOLANDA LS, et al. Evolução Hospitalar de Pacientes com Endocardite Infecciosa em Hospital Público de Belém, Pará - Brasil. International Journal of Cardiovascular Sciences, Belém, PA - Brasil, 2015; 28(6): 496-503.

14. HOLANDA LS, et al. Hospital evolution of patients with infective endocarditis in a public hospital in Belém, Pará, Brazil. International Journal of Cardiovascular Sciences, 2016; 28(1).

15. HOLLAND TL, et al. Infective Endocarditis. Nature Reviews Disease Primers. 2016; 16059.

16. HUBERS SA, et al. Infective Endocarditis: A Contemporary Review. Mayo Clin Proc, 2020; 95(5): 982-997.

17. KARCHMER, A. W. et al. Braunwald's heart disease: a textbook of cardiovascular medicine. Philadelphia, US: Elsevier Saunders, 2012; 9.

18. LAINSCAK M, et al. Guidelines for the management of infective endocarditis. European Heart Journal. ESC, 2015; 36: 3075-3123.

19. LAMAS CC. Endocardite infecciosa: Ainda uma doença mortal. Arquivos Brasileiros de Cardiologia, 2020; 114(1): 911.

20. LIESMAN RM, et al. Laboratory diagnosis of infective endocarditis. Journal of clinical microbiology, 2017; 55(9): 25992608.

21. LOMAS JM, et al. Healthcare-associated infective endocarditis: an undesirable effect of healthcare universalization. Clin Microbiol Infect. 2010; 16(11): 1683-90.

22. MACHADO FCA, FERREIRA MAF. Perfil da endocardite infecciosa em hospital de referência entre 2003 e 2009 . Rev. Bras. Odontol., Rio de Janeiro, 2013; 70(1): 8-11.

23. MADEIRA MP. Perfil dos pacientes internados com diagnóstico de endocardite infecciosa em serviço de Clínica médica de um hospital terciário do Ceará. Dissertação (Monografia de final de residência médica em clínica médica) - Hospital Geral de Fortaleza. Escola de Saúde Pública do Ceará, Fortaleza, 2018; 32p.

24. MARQUES A, et al. Fatores de Risco para Mortalidade Hospitalar na Endocardite Infecciosa. Arquivos Brasileiros de Cardiologia, 2019; 114: 1-8.

25. PINHEIRO IL, et al. Endocardite infecciosa em cirurgias valvares: avaliação ecocardiográfica e clínica como preditores de mortalidade em uma série de casos. Rev Soc Bras Clin Med; 2018; 16(2): 113-115.

26. SARAIVA MGG, et al. Epidemiology of infectious meningitis in the State of Amazonas, Brazil. Revista da Sociedade Brasileira de Medicina Tropical, 2015; 48: 79-86.

27. SOBREIRO DI, et al. Diagnóstico Precoce da Endocardite Infecciosa: Desafios para um Prognóstico Melhor. 2019.

28. TARASOUTCHI F, et al. Diretriz Brasileira de Valvopatias - SBC 2011/I Diretriz Interamericana de valvopatias- SIAC 2011. Arquivos Brasileiros de Cardiologia, 2011; 97: 01-67.

29. VIEIRA PM, et al. Endocardite infecciosa em cabo de marcapasso por Enterobacter cloacae: Relato de Caso. Revista de Saúde, 2020; 11(2):33-38.

30. WANG A, et al. Management Considerations in Infective Endocarditis: A Review. JAMA, 2018; 320(1):72-83.

31. ZIPES DP, et al. Braunwald: Tratado de doenças cardiovasculares. Rio de Janeiro: Elsevier, 2013; 9. 